\title{
Protein kinase E of Mycobacterium tuberculosis has a role in the nitric oxide stress response and apoptosis in a human macrophage model of infection
}

\author{
Deepak Jayakumar, ${ }^{1 \dagger}$ William R. Jacobs $\mathrm{Jr}^{2}$ and \\ Sujatha Narayanan ${ }^{1 *}$ \\ ${ }^{1}$ Department of Immunology, Tuberculosis Research \\ Centre (ICMR), Chetput, Chennai-600031, India. \\ ${ }^{2}$ Department of Microbiology and Immunology, Albert \\ Einstein College of Medicine, Bronx, New York, USA.
}

\section{Summary}

Mycobacterium tuberculosis, an intracellular pathogen, inhibits macrophage apoptosis to support survival and replication inside the host cell. We provide evidence that the functional serine/threonine kinase, PknE, is important for survival of M. tuberculosis that enhances macrophage viability by inhibiting apoptosis. A promoter of PknE identified in this study was shown to respond to nitric oxide stress. Deletion of pknE in virulent $M$. tuberculosis, H37Rv, resulted in a strain that has increased resistance to nitric oxide donors and increased sensitivity to reducing agents. The deletion mutant created by specialized transduction induced enhanced apoptosis while inhibiting necrosis. The pknE mutant also modifies the innate immune response as shown by the marked decline in the pro-inflammatory cytokines in a macrophage model of infection. These findings suggest a novel mechanism, by which PknE senses nitric oxide stress and prevents apoptosis by interfering with host signalling pathways.

\section{Introduction}

Mycobacterium tuberculosis causes 8 million new cases and continues to kill at least 2 million people per year (Kaufmann and McMichael, 2005). In a vast majority of infected persons, the pathogen is not eliminated but lies dormant in the foci of chronic granulomatous tissues. Available genetic tools have enabled researchers to dissect and decipher the function of genes that play a role

Received 31 July, 2007; revised 24 August, 2007; accepted 30 August, 2007. *For correspondence. E-mail sujatha.sujatha36@ gmail.com; Tel. (+91) 44 28369627, ext 4456; Fax (+91) 44 28362528. 'Present address: Department of Biological Engineering, State University of New York (SUNY) at Buffalo, New York, USA. in the pathogenesis of tuberculosis (TB). Although several genes are being implicated in the pathogen's propensity to cause disease and persist in host tissues, the exact mechanisms that aid the tubercle bacillus to reside, multiply and/or remain latent within host cells are poorly understood.

Serine threonine protein kinases are signal transduction molecules that play important roles in the physiology and virulence of $M$. tuberculosis. However, till date, only $\mathrm{PknG}$ and $\mathrm{PknH}$ have been implicated in these processes (Cowley et al., 2004; Walburger et al., 2004; Papavinasasundaram et al., 2005). PknA, PknB and Pknl have been linked to the physiological process of cell elongation/ division (Kang et al., 2005; Singh et al., 2006). We had delineated the promoter of PknE, and we have seen that it is regulated under nitric oxide stress condition. The PknE promoter sequence showed increased activity when the cells were exposed to nitric oxide donors. This prompted us to explore the role of PknE in the intracellular survival of $M$. tuberculosis because nitric oxide is intimately linked with host cell apoptosis.

There is ample evidence to suggest that apoptosis or programmed cell death is an important mechanism associated with M. tuberculosis infection (Danelishvili et al., 2003). The induction of apoptosis could contribute to protection by directly killing the mycobacteria or by strengthening the adaptive immunity. Apoptosis is therefore regarded as a critical defence strategy to slow down the dissemination of bacteria inside the host. Inhibition of macrophage apoptosis by bacterial pathogens constitutes a significant virulence mechanism in the context of $M$. tuberculosis pathogenesis, because it allows the pathogen to survive and replicate in live host cells.

Eukaryotic kinases such as PKR have been linked to apoptosis of host cells and innate immunity (Cheung et al., 2005). However, bacterial components linked to apoptosis are poorly understood, and the exact mechanisms underlying their interaction with host signalling elements are not known. Therefore, there is paucity in literature concerning the roles of mycobacterial genes preventing apoptosis, when tubercle bacilli reside inside host cells. Only two serine/threonine kinases of mycobacteria, namely PknG and $\mathrm{PknH}$, have been shown to exhibit virulence modifying properties in a murine model of infection (Cowley et al., 
2004; Papavinasasundaram et al., 2005). Additionally, PknG inhibited phagosome lysosome fusion in Mycobacterium bovis BCG (Walburger et al., 2004).

Nitric oxide is a diffusible, multifunctional transcellular messenger that has been implicated in numerous physiological and pathological states, including mycobacterial pathogenicity (Chan et al., 2001; Visca et al., 2002). There are several reports documenting the importance of apoptosis and the vital role of nitric oxide in this process (Brüne et al., 1998, Chung et al., 2001; Choi et al., 2002). There is also experimental evidence to suggest that low levels of nitric oxide ( $<100$ p.p.m.) can result in the effective intracellular killing of $M$. tuberculosis up to $99 \%$ (Long et al., 1999) by several potential mechanisms. As our data strongly suggest that $p k n E$ has a vital role in the nitric oxide stress response, this finding, along with previous studies of nitric oxide and apoptosis, led us to further explore the role of $p k n E$ in macrophage viability and also to establish a potential link between the nitric oxide stress response and host cell apoptosis.

We provide evidence for the first time in this report that deletion of a serine/threonine kinase, pknE, of $M$. tuberculosis results in increased apoptosis of an in vitro human macrophage model of infection. Further, the pknE mutant-infected cells show a decrease in production of pro-inflammatory cytokines, TNF- $\alpha$ and interleukin (IL)-6. This report also shows that the pknE-deletion mutant exhibits in vitro resistance to nitric oxide donors and increased nitric oxide-mediated apoptosis in human macrophages. Therefore, this study provides new insights into the anti-apoptotic role of this stress-responsive signalling element in the pathogenesis of $M$. tuberculosis.

\section{Results}

The promoter of the pknE gene responds to nitric oxide stress, mediated by the nitric oxide donors

The promoter activity of $p E-U P S 1$, shown by expression of $\beta$-galactosidase, was differentially regulated under oxidative stress and nitrosative stress (Fig. 1). A marked reduction in the promoter activity was seen when subjected to oxidative stress (hydrogen peroxide) even after $0.5 \mathrm{~h}$ of exposure, probably suggesting that the expression of this gene may not be necessary during oxidative stress. On the other hand, $\beta$-galactosidase activity was upregulated by 0.5-fold after $2 \mathrm{~h}$ exposure to the nitric oxide donor, sodium nitroprusside (SNP). Similar upregulation of promoter activity (146 Miller units) after $2 \mathrm{~h}$ was seen when another nitric oxide donor, S-nitrosoglutathione (GSNO) (final concentration $=1 \mathrm{mM}$ ), was used. No significant differences were recorded in the viability counts of cells subjected to stress when compared with the pE-UPS1 control, suggesting that the observed levels of promoter activity

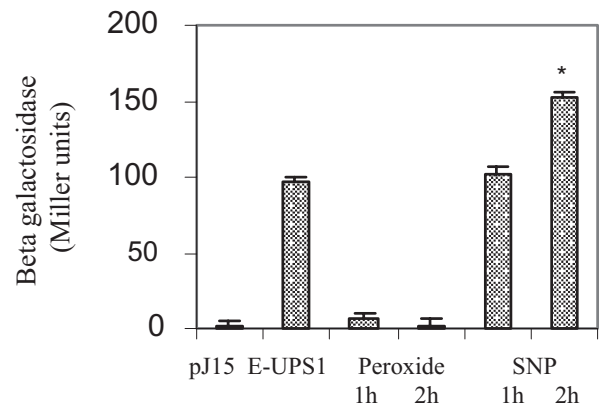

Control, hydrogen peroxide and SNP (NO donor) induced strains

Fig. 1. $\beta$-Galactosidase assay. The $p k n E$ promoter is induced under nitric oxide stress and is repressed under oxidative stress in M. smegmatis. Hydrogen peroxide $\left(\mathrm{H}_{2} \mathrm{O}_{2}\right)$ was used for oxidative stress, and sodium nitroprusside (SNP) was used for nitrosative stress. Briefly, the upstream sequence of $p k n E$ was cloned in a promoter probe vector, and the construct ( $p E-U P S 1)$ was introduced into $M$. smegmatis. Promoter activity was monitored using the $\beta$-galactosidase assay, and the results are represented in Miller units. pJ15 and E-UPS1 are the control values; 1 or $2 \mathrm{~h}$ exposure of E-UPS1 to $10 \mathrm{mM} \mathrm{H}_{2} \mathrm{O}_{2} ; 1$ or $2 \mathrm{~h}$ exposure of E-UPS1 to $10 \mathrm{mM}$ SNP. ${ }^{*} P<0.05$

were the result of the response to the different agents used and not because of altered viability of the bacteria used for the experiments.

The pknE mutant of $\mathrm{M}$. tuberculosis is resistant to nitric oxide donors and sensitive to reducing agents and certain metal ions

The pknE-deletion mutant which we constructed by specialized transduction (Fig. S1 and Table S1) was not different from the wild type when in vitro growth profiles were compared or when tested in vitro with glutamine synthetase inhibitors, L-methionine sulfoximine and DL-Phosphinothricin, and different concentrations of sodium chloride $-50,100,250$ and $500 \mathrm{mM}$ (data not shown). The $\Delta p k n E$ mutant was sensitive to hydrogen peroxide when compared with the wild-type strain, but this difference was not statistically significant (data not shown). On the other hand, the mutant was resistant to varying degrees to nitric oxide donors (SNP, GSNO and acidified nitrite) added to in vitro-grown cultures of the wild-type, mutant and complemented strains (Fig. 2). The mutant also exhibited marked sensitivity to dithiothrietol (DTT), reduced glutathione (GSH), zinc and cadmium (Fig. 3), but not to mercury or iron (data not shown).

The pknE mutant of M. tuberculosis increases apoptosis in a human macrophage model of infection, including nitric oxide-mediated apoptosis

Three assays were performed in this study to ascertain the role of $p k n E$ in apoptosis of differentiated primary 


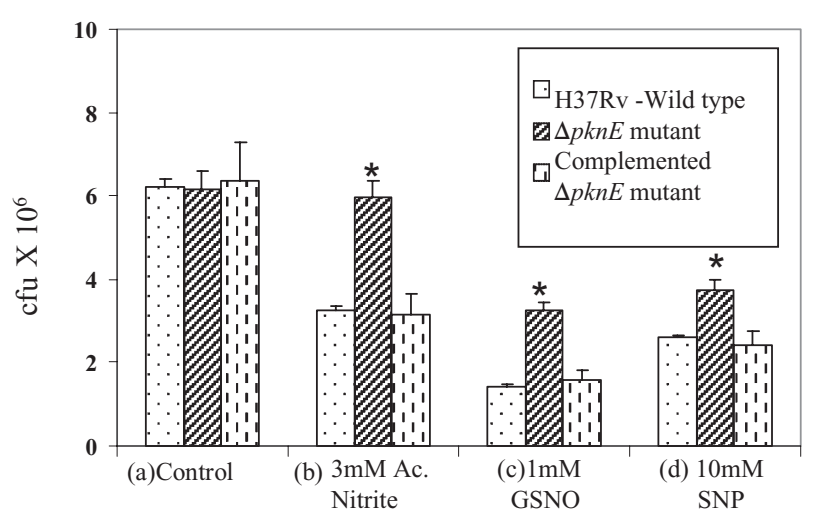

Fig. 2. Sensitivity of the $\triangle p k n E M$. tuberculosis mutant to nitric oxide donors: (a) control-untreated; (b) $3 \mathrm{mM}$ acidified nitrite, pH 5.4; (c) $1 \mathrm{mM}$ GSNO; and (d) $10 \mathrm{mM}$ SNP. The three strains were treated in triplicates, and cells were harvested, diluted and plated on $7 \mathrm{H} 10$ plates. The results were represented graphically \pm SEM. ${ }^{*} P<0.05$
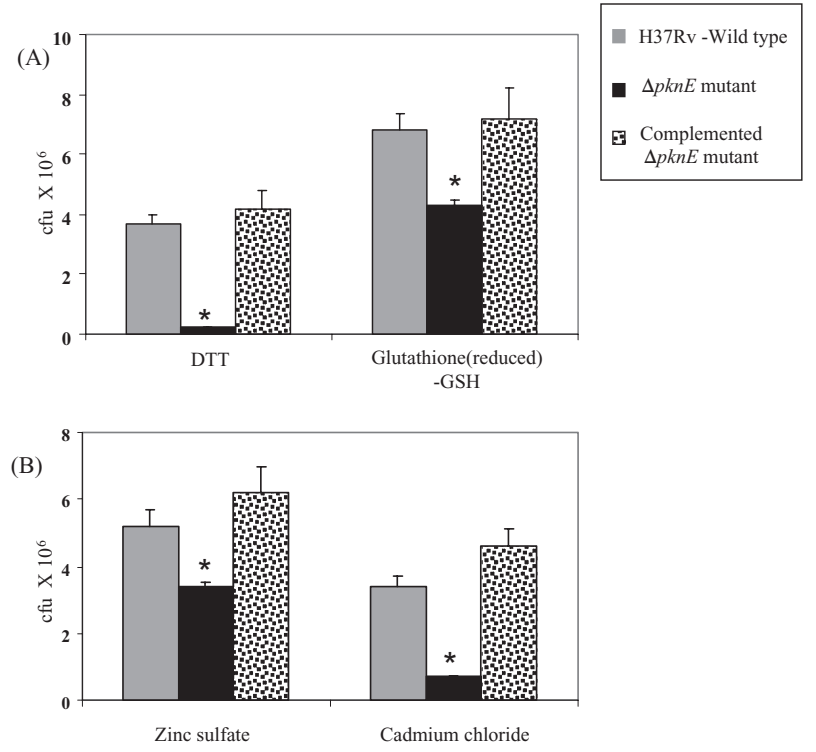

Fig. 3. Sensitivity of the M. tuberculosis $\Delta p k n E$ mutant to reducing agents and divalent cations.

A. DTT (Cleland's reagent) $12.5 \mu \mathrm{M}$ and GSH (reduced GSH $10 \mu \mathrm{M}$ (reducing agents)].

B. Metal ions: zinc and cadmium, $100 \mu \mathrm{M}$ each. Values indicated are final concentrations. The three strains of $M$. tuberculosis were incubated in $7 \mathrm{H} 9$ broth with the above reagents, and cfu were determined after $48 \mathrm{~h}$ exposure and plating each culture in triplicates. ${ }^{*} P<0.05$ human macrophages. Consistent results were obtained which clearly showed that $p k n E$ mutant-infected macrophages exhibited increased apoptosis when compared with wild-type and complemented strain-infected cells. Increase in macrophage apoptosis following infection was observed after annexin V/propidium iodide staining and fluorescence-activated cell sorting (FACS) analysis after day 1 (Fig. S2), day 3, day 5 and day 7 (data not shown) post infection. Terminal deoxynucleotidyl transferase biotin-dUTP nick end-labelling (TUNEL) staining and FACS analysis confirmed increases in apoptosis at all time points of infection (Fig. 4), where percentage positive cells were statistically increased in mutant-infected macrophages. Additionally, DNA extracted from pknE mutant-infected macrophages exhibited marked fragmentation (data not shown). Apoptosis observed in mutantinfected macrophages was correlated to a decline in GSH content in macrophages (Fig. S3).

The annexin V/propidium iodide assay and the TUNEL assay were repeated after treating the infected primary human macrophages with nitric oxide donor, SNP. Increased apoptosis (Fig. 5), and more than a twofold increase in the percentage of propidium iodide-positive cells, were seen in the $\Delta p k n E$ mutant-infected macrophages in comparison with the control (uninfected), wildtype and complemented strain-infected macrophages after exposure to $10 \mathrm{mM}$ of SNP for $3 \mathrm{~h}$ (Table 1).

The pknE mutant of M. tuberculosis inhibits two important cellular events, necrosis and proliferation in macrophages

Lactate dehydrogenase (LDH) release assays were performed to assess necrosis from supernatants collected from the macrophage-infected cultures (Fig. 6). The levels of $\mathrm{LDH}$ were significantly lower in the case of mutantinfected macrophages at all the time points of infection $(P<0.05)$, showing that necrosis of infected macrophages was significantly reduced. Glycine protection assays (Fig. 6B) revealed that $5 \mathrm{mM}$ of glycine reduced the levels of LDH in infected cultures, implying that this compound partially protected the cells from necrosis. Reduced viability of mutant-infected macrophages was observed by the trypan blue dye exclusion assay and the MTT

Table 1. pknE mutant-infected macrophages show increased secondary necrosis after exposure to nitric oxide.

\begin{tabular}{llcc}
\hline Strain no. & Strain used to infect THP-1 macrophages & Control $(3 \mathrm{~h})$ & $10 \mathrm{mM} \mathrm{SNP}(3 \mathrm{~h})$ \\
\hline 1 & M. tuberculosis, H37Rv & $4.5 \pm 1.5$ & $8.41 \pm 1.5$ \\
2 & $\Delta$ pknE mutant M. tuberculosis & $6.0 \pm 2.1$ & $14.68 \pm 3.8^{*}$ \\
3 & Complemented $\Delta$ pknE mutant & $3.1 \pm 2.4$ & $9.07 \pm 1.7$ \\
\hline
\end{tabular}

Percentage of propidium iodide-positive cells by FACS analysis after exposure of infected cells to nitric oxide donor, SNP. Results shown here are the average of three independent experiments.

${ }^{*} P<0.05$. 
(A)

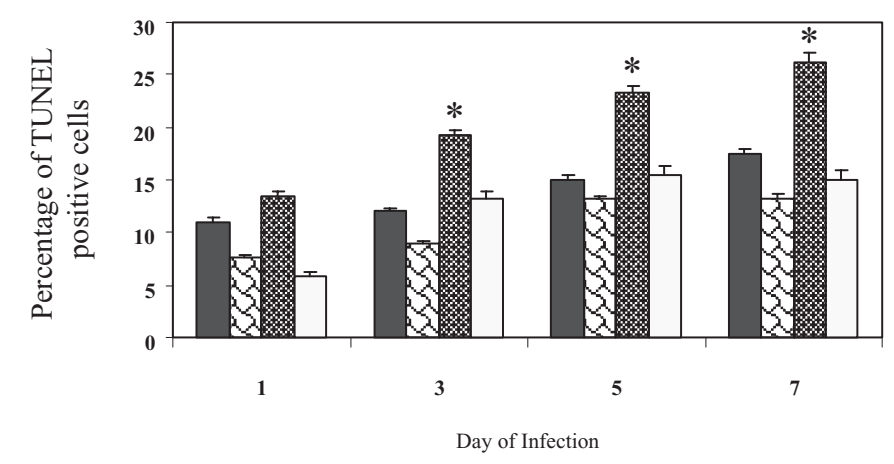

Fig. 4. TUNEL assay. Confirmation of apoptosis by the pknE mutant of M. tuberculosis.

A. Values were found to be statistically significant $\left({ }^{*} P<0.05\right)$. The results presented are the averages of three independent experiments \pm SEM.

B. Representative quadrant analysis of TUNEL-positive populations in THP-1 macrophages infected with the three strains on day 5 of infection. Percentages are indicated in the lower right quadrant of every plot.
(B)

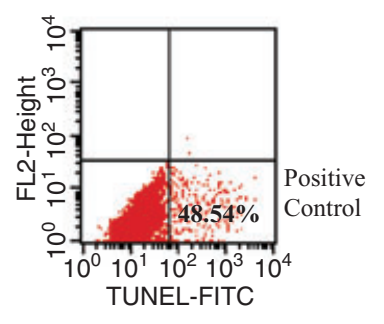

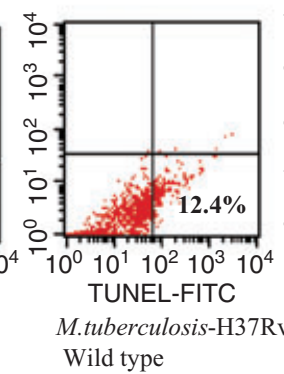

Wild type

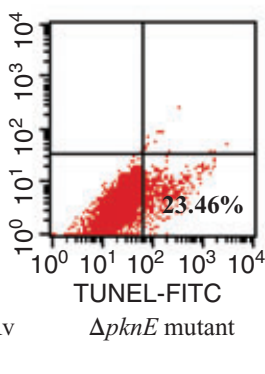
assay (Fig. S4). Proliferation assays were conducted with
infected cells using both $\mathrm{H}^{3}$-thymidine incorporation assay (data not shown) and proliferating cell nuclear antigen (PCNA) staining, followed by FACS analysis (Fig. 7). Decrease in the level of proliferation as measured by counts per minute of mutant-infected cells, and a statistically significant downregulation of the PCNA intracellular marker in the mutant-infected macrophages, clearly demonstrated that a majority of the mutant-infected cells were in various stages of apoptosis.

The pknE mutant of M. tuberculosis exhibits an altered intramacrophage survival and pro-inflammatory cytokine response in a THP-1 model of infection

Although no differences are observed in the earlier time points, the mutant-infected macrophages yield lesser bacilli during the later time points of infection (Fig. S5). A

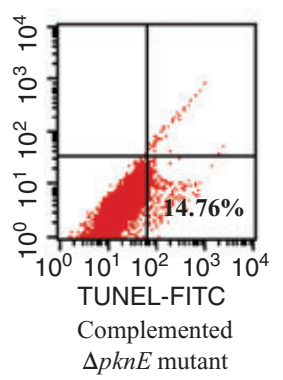

marked reduction (at least 8- to 10-fold) in the cytokines TNF- $\alpha$ and IL- 6 was observed at all the time points tested (Fig. 8A and $\mathrm{B}$ ) in pknE mutant-infected macrophages. The results were found to be statistically significant $(P<0.05)$. No significant differences were seen in secretion of IFN- $\gamma$, IL-4, IL-10 and IL-12p70 (data not shown).

\section{Discussion}

After the release of the complete genome sequence of M. tuberculosis (Cole et al., 1998), there is increasing evidence that serine/threonine kinases are candidate elements that play a key role in the physiology and pathogenesis of $M$. tuberculosis. In this report, we elaborate significant findings on PknE, whose function was largely unknown although a recent report suggests that PknE is one of the kinases that phosphorylates an anti-anti-sigma factor homologue (Greenstein et al., 2007). We chose this 


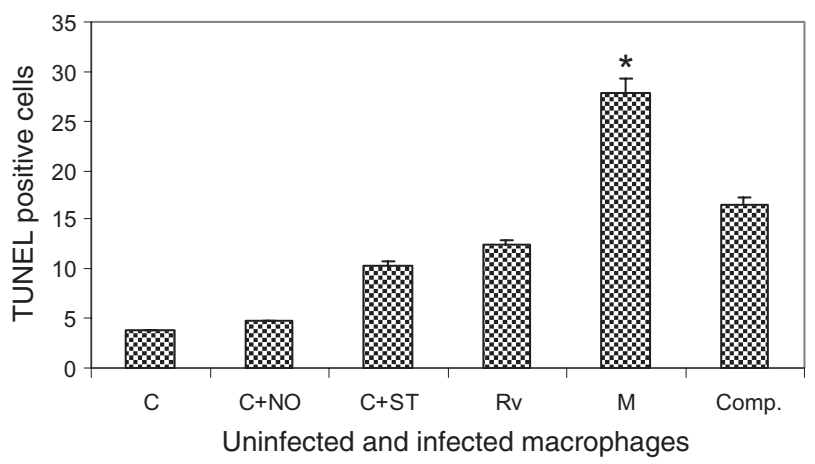

Fig. 5. TUNEL assay with nitric oxide. TUNEL assay was conducted for infected primary human macrophages infected with wild-type, mutant and complemented strains exposed to $10 \mathrm{mM}$ SNP for $2 \mathrm{~h}$. Without nitric oxide treatment, the numbers of TUNEL-positive cells were as follows: wild type, $6.2 \%$; mutant $7.4 \%$; and complemented strain, $5.3 \%$. The results presented here are representative of three experiments \pm SEM $\left({ }^{*} P<0.05\right)$, where the $y$-axis denotes percentage of TUNEL-positive cells. C, uninfected control cells; C + NO, control cells with nitric oxide donor, SNP; C + ST, staurosporine-treated cells; Rv, cells infected with H37Rv; M, cells infected with the pknE mutant; Comp., cells infected with the complemented $p k n E$ mutant.

kinase because our initial studies with the $p k n E$ promoter suggested that this gene plays a key role in the response to nitric oxide. Also, in silico amino acid sequence analysis indicated a DSBA-like thioredoxin domain at the $\mathrm{C}$-terminus of the protein. This kinase has been biochemically characterized (Molle et al., 2003) and has been predicted to transduce signals from the macrophage environment to the cell interior based on its topology and predicted membrane organization. This study highlights evidence that it plays a key role in apoptosis and immune response in a macrophage model of infection. As a first step in this study, we decided to identify the possible factors that could trigger the elevated expression of this enzyme. The promoter of this signalling element was found to lie upstream to the open reading frame coding for $p k n E$.

This promoter sequence when cloned in a promotertrap vector and electroporated into the saprophytic strain, Mycobacterium smegmatis, showed enhanced expression of the reporter gene ( $\beta$-galactosidase activity) when subjected to nitrosative stress via nitric oxide donors. These results were concordant with findings of Voskuil et al. (2003), where microarray analysis of gene expression indicated that $p k n E$ was one among the several genes that showed enhanced expression under nitric oxide stress. The products of genes that respond to stress can make the bacterium either more susceptible or resistant to the action of nitric oxide.

In order to address the significance of this finding, a $p k n E$ gene-deficient strain and the corresponding complemented strain of the pknE mutant were constructed in M. tuberculosis. The pknE mutant exhibited a marked resistance to nitric oxide donors, which was statistically significant when compared with the wild-type strain, and this result was in accordance with that reported for another kinase, $p k n H$ (Papavinasasundaram et al., 2005). Hence, it is possible that both PknE and PknH are enzymes that scavenge nitric oxide-free radicals from the intracellular environment and make the organism more sensitive to this innate defence mechanism. Another interesting observation that was in accordance with our in silico analysis of the primary PknE protein sequence was the sensitivity of the $p k n E$ mutant to reducing agents. This property could be attributed to the loss of the C-terminal DSBA-like thioredoxin domain with the CXXC motif that has been reported for other pathogenic bacteria (Tinsley et al., 2004). It has been reported that mutations in $d s b A$ and $d s b B$ but not $d s b C$ confer sensitivity to mercury and cadmium in Escherichia coli (Stafford et al., 1999). This finding also raises the possibility that PknE might play a role in redox signalling/homeostasis within the cell, after responding to stress. However, mere possession of the DSBA domain does not confer chaperone/isomerase functions to the protein, as a considerable gain of function

(A)

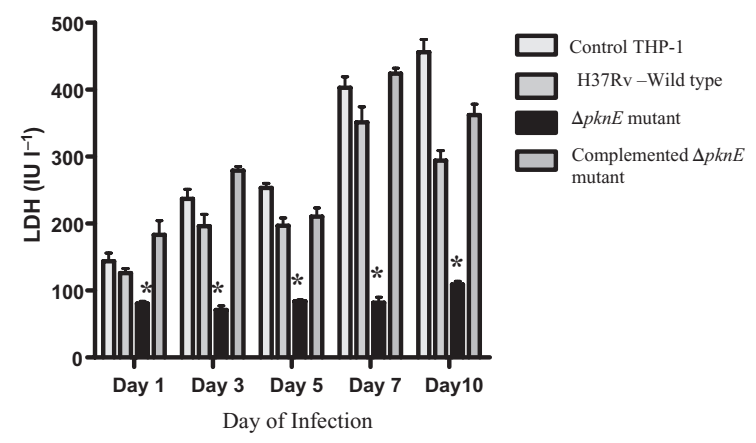

(B)

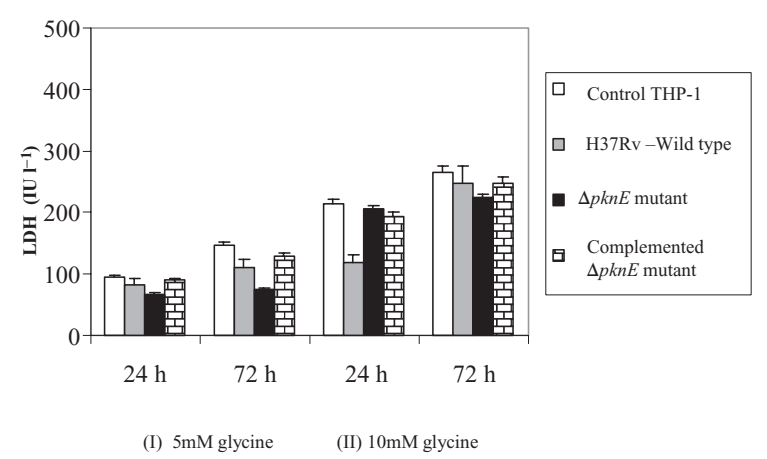

Fig. 6. LDH release assay.

A. Culture supernatants from THP-1 cells infected with the above strains were assayed for human LDH. Results shown are the average of triplicate experiments \pm SEM $\left({ }^{*} P<0.05\right)$. Glycine protection assay (B), in which (I) $5 \mathrm{mM}$ and (II) $10 \mathrm{mM}$ glycine have been added to the cultures. Addition of glycine partially protected the cultures against necrosis. 
(A)

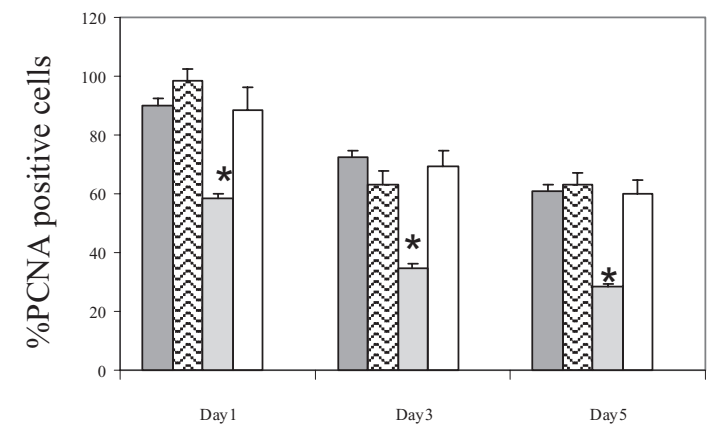

Day of Infection
Fig. 7. PCNA assay. PCNA staining of THP-1 macrophages. THP-1 cells infected with the above three strains were analysed by flow cytometry. Graphical representation of percentage of PCNA stained cells are indicated above in (A). Values between wild-type and mutant were statistically significant $\left({ }^{\star} P<0.05\right)$. Representative histograms of THP-1 cells stained for PCNA on day 3 of infection (B), where M1 indicates the unstained population and $\mathrm{M} 2$ represents PCNA-stained population.

(B)

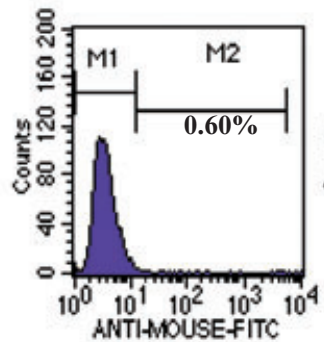

THP-1 FITC conjugate Control

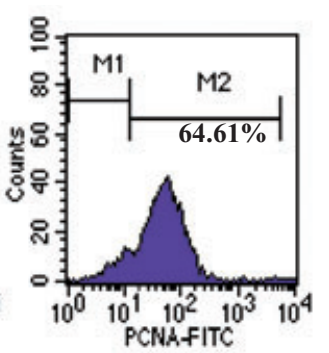

THP-1 stained for PCNA

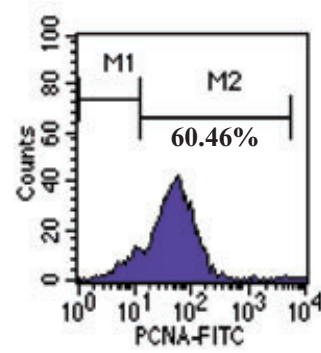

M.tuberculosis-H37Rv Wild type

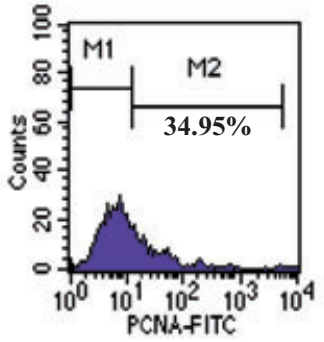

$\Delta p k n E$ mutant

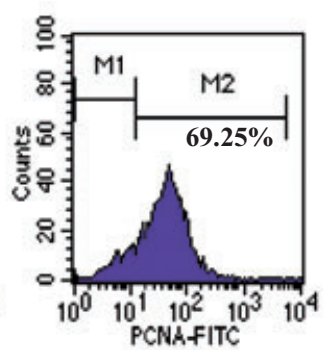

Complemented $\Delta p k n E$ mutant is achieved by dimerization with other disulfide bondcontaining proteins (Zhao et al., 2003). Functional characterization of the DSBA domain is essential to ascertain its exact physiological role.

The pknE mutant of M. tuberculosis exhibited lower rates of survival and multiplication in an in vitro macrophage model of infection, which is consistent with the results obtained for the pknE mutant used in mouse-infection studies reported earlier (Papavinasasundaram et al., 2005) where lower numbers of bacilli were recovered from the organs of pknE mutant-infected mice. However, these experiments have their own limitations because in vitrogrown macrophages do not exactly simulate the conditions that exist in host tissues and the mouse is not an ideal model to evaluate the pathogenicity of M. tuberculosis due to differences in tuberculous pathology between mice and humans.

The pknE gene-disrupted strain also significantly enhanced macrophage apoptosis, and it was confirmed in this study that apoptosis was the predominant mechanism of death in macrophages. Increased apoptosis induced by the pknE mutant was not a THP-1 macrophage cell-linerestricted event, as it was also observed at comparable levels in primary human macrophages. A simultaneous decline in reduced GSH in mutant-infected macrophages further augmented the finding that the mutant-infected macrophages exhibited reduced viability. Because the level of apoptosis was high in the mutant-infected macrophages, coincidentally, levels of necrosis and proliferation were low. This study also demonstrates for the first time the association between PCNA positivity and mycobacterial infection. There are specific components of virulent $M$. tuberculosis, such as LAM, the $19 \mathrm{kDa}$ lipoprotein or the ESAT-6 protein that can induce apoptosis of infected macrophages (Lopez et al., 2003; Dao et al., 2004; Derrick and Morris, 2007). On the other hand, there are genes such as $n l a A$ and $\operatorname{sod} A$ that reduce apoptosis of mycobacterium-infected cells (Skeiky and Sadoff, 

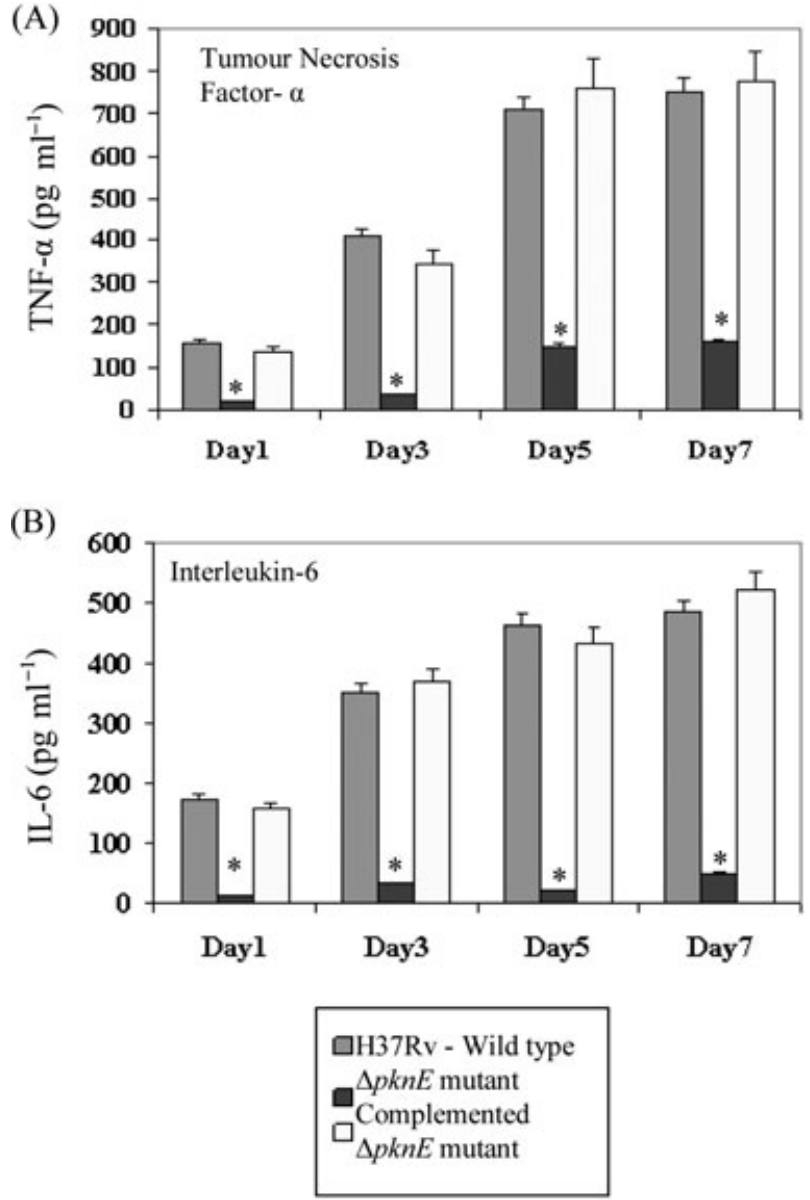

Fig. 8. Enzyme-linked immunosorbent assay (ELISA) for TNF- $\alpha$ and IL-6. Cytokine estimation for culture supernatants infected with H37Rv (wild type), pknE mutant and the complemented strains. The protocol was the same as prescribed by the manufacturer based on the principle of a sandwitch ELISA. The capture antibody was coated on the ELISA plate; the samples were added, followed by the detection antibody. The samples were read by a spectrophotometer after the addition of the substrate. The figures show (A) TNF- $\alpha$ and (B) IL-6 levels at different time points of infection. The data shown above are the averages of triplicate values \pm SEM $\left({ }^{*} P<0.05\right)$.

2006) in addition to nuoG of M. tuberculosis (Velmurugan et al., 2007). Therefore, a delicate balance exists between the apoptosis-inducing and the apoptosis-evading components, and this balance decides the survival of the pathogen within host cells. A direct beneficial implication of increased apoptosis and less necrosis of macrophages induced by the pknE mutant would be efficient intracellular killing and better antigen cross-presentation to eliminate the bacilli from the host.

Another intriguing result was that the addition of nitric oxide donors to in vitro-grown macrophages induced increased apoptosis of mutant-infected macrophages, which rapidly progressed to secondary necrosis. This strongly suggests the cytoprotective role of PknE in indi- rectly preventing apoptosis of infected macrophages, specifically when exposed to nitric oxide stress. Recently, inhibition of apoptosis has been intimately linked with nitric oxide detoxification mechanisms in Neisseria sp. (Tunbridge et al., 2006). Hence, in this organism failure of apoptosis induction would lead to high-level expression of pro-inflammatory cytokines, and tissue injury. In M. tuberculosis, however, deletion of pknE resulted in in vitro resistance to nitric oxide donors but led to an induction of apoptosis and loss of the pro-inflammatory cytokine response. This clearly shows that PknE is a possible anti-apoptotic regulator but also plays a role in susceptibility of the bacilli to nitric oxide. Therefore, the nitric oxide stress response and induction of apoptosis are closely associated with each other with respect to the pknE mutant. It can be hypothesized that PknE senses nitric oxide from the environment and possibly interacts with other mycobacterial and/or host cell components, finally resulting in evasion of apoptosis. Incidentally, we also showed that recombinant PknE expressed in E. coli cross-reacts with phospho-SAPK/JNK antibody, suggesting similarities in function (capable of responding to stress) and amino acid sequence.

Significant reduction in the secretion of the proinflammatory cytokines, namely TNF- $\alpha$ and IL-6, was observed in the culture supernatants of the mutantinfected THP-1 macrophages. Deletion of either specific genes (Copenhaver et al., 2004; Rao et al., 2005) or operons (Shimono et al., 2003; Cheruvu et al., 2007) has led to a decrease in these pro-inflammatory cytokines. Increased necrosis, high TNF- $\alpha$ levels and reduced IL-6 have been reported for macrophages infected with the virulent clinical isolate of $M$. tuberculosis, CSU22 (Park et al., 2006). Our results show that PknE might indirectly boost the innate immune response, thereby restricting the growth and survival of bacilli in the intracellular environment. However, a major limitation to this experiment would be that it does not mimic the cytokine response that would normally occur within infected host tissues. Hence, cytokine responses can be more reliably delineated and interpreted by performing co-culture experiments or estimating cytokines in sera collected from infected animals. This study essentially highlights apoptosis and antiinflammation that occurs when a key sensory molecule is deleted from pathogenic $M$. tuberculosis. This is in accordance with a recent report in the context of bacterial flagellin, which is a microbial-associated molecular pattern, a potent inducer of inflammatory responses and an antiapoptotic regulator (Neish, 2007).

The results of this study indicate that serine/threonine kinases are key regulatory molecules that are not only essential for the intracellular survival of the slow-growing pathogen, M. tuberculosis, but also play a role in the stress response of the pathogen. The $\Delta p k n E$ mutant of 
M. tuberculosis displays multiple phenotypes within the intracellular environment, and hence this gene along with others may be important for the pathogenesis of TB.

\section{Experimental procedures}

\section{Cloning of the pknE promoter and expression} in M. smegmatis

A $9.3 \mathrm{~kb}$ promoter probe, operon fusion vector pJEM15 (Timm et al., 1994), which has a lacZ reporter gene coding for the $\beta$-galactosidase enzyme, was used for cloning purposes. Both E. coli $\mathrm{DH} 5 \alpha$ and $M$. smegmatis $\mathrm{mc}^{2} 155$ (Table S1B) recombinant strains were maintained in Luria-Bertani kanamycin $\left(25 \mu \mathrm{g} \mathrm{ml}^{-1}\right)$ agar plates. Primers E8671 and E9215c (Table S1A) were used to amplify the $545 \mathrm{bp}$ upstream sequence of $p k n E$. The PCR product was then cloned into pTOPO vector (Invitrogen, USA), subcloned into pJEM15, and the sequence was verified by automated sequencing. This construct was electroporated into $M$. smegmatis, and the $\beta$-galactosidase assay was carried out according to Miller (1972).

\section{Determination of sensitivity/resistance to various compounds for the pknE mutant of $\mathrm{M}$. tuberculosis}

The pknE mutant of $M$. tuberculosis was tested for its sensitivity to various compounds, which included the: (i) glutamine synthetase inhibitors, L-sulfoximine and DL-Phosphinothricin (Sigma, USA); (ii) salt; (iii) nitric-oxide donors [SNP (Sigma), GSNO (Calbiochem, USA), and acidified nitrite]; (iv) hydrogen peroxide; (v) metallic salts-cadmium chloride, zinc sulfate, iron sulfate and mercuric chloride; (vi) reduced GSH (Calbiochem, USA); and (vii) DTT at specified concentrations.

\section{Terminal deoxynucleotidyl transferase biotin-dUTP nick end-labelling (TUNEL) assay and FACS analysis}

To detect the DNA fragmentation, the TUNEL procedure was applied, using the In Situ Cell Death Detection Kit (Roche Diagnostics, Mannheim, Germany) according to the manufacturer's instructions. The cells fixed with $4 \%$ paraformaldehyde were briefly washed twice with PBS and then stained with the TUNEL reaction mixture. The cells were then incubated at $37^{\circ} \mathrm{C}$ for $1 \mathrm{~h}$ before acquisition and analysis using the BD FACSCalibur flow cytometer. This experiment was repeated thrice, and a positive control (staurosporine-treated THP-1 culture) was included in every experiment.

\section{Proliferating cell nuclear antigen (PCNA) staining and FACS analysis}

Proliferating cell nuclear antigen (PCNA)-10 staining was effected using an indirect method. The primary anti-mouse PCNA-10 antibody was purchased from Dako Corporation, USA. The secondary antibody was a goat anti-mouse FITC conjugate, purchased from Bangalore Genei, India. Fixation/ permeabilization buffer and wash buffer from the intracellular human cytokine starter kit (BD Pharmingen, USA) was used for the staining protocol. The cells were infected with the strains and fixed with $4 \%$ paraformaldehyde. For the PCNA staining, the cells were fixed and permeabilized using the human cytokine starter kit and stained with primary antibody overnight at 1:1000 dilution. The cells were washed twice with wash buffer (centrifuged at 10000 r.p.m. for $10 \mathrm{~min} \times 2$ ) and stained with the secondary conjugate for $2 \mathrm{~h}$ at 1:800 dilution. The cells were washed twice again with permeabilization buffer and were acquired and analysed using the BD FACSCalibur flow cytometer. This experiment was repeated thrice and the results are presented with \pm SEM.

\section{Lactate dehydrogenase assay}

Lactate dehydrogenase (LDH) assays were conducted using the ACE $^{\text {TM }}$ LDH-P Reagent from Alfa Wassermann, the Netherlands, and the $A C E^{\mathrm{TM}}$ clinical chemistry system. The readings were taken using a clinical chemistry autoanalyser. The LDH assays were performed in triplicate for each sample and were also repeated for the glycine protection assay. The values were expressed in international units per litre. The expected values in the range of $225-450 \mathrm{U} \mathrm{I}^{-1}$ were detected in supernatants harvested from uninfected THP-1 cells.

\section{Enzyme-linked immunosorbent assay}

The supernatants of the infected cell lines (infected with the wild-type, mutant and complemented strains) were collected for each time point, and cytokines such as TNF- $\alpha$, IFN- $\gamma$, IL-10 and IL-12p70 (from R \& D, USA) and IL-6 (from BD Pharmigen, USA) were estimated in triplicates using commercially available kits. Each assay was run with a negative control (PBS) and blank samples. The average zero standard optical density was subtracted from different standard and optical densities. Sample concentrations were calculated using a standard curve. The results were expressed as $\mathrm{pg} \mathrm{ml}^{-1}$.

\section{Acknowledgements}

D.J. was supported by the AECOM AIDS International Training and Research Program (D43 TW 001403; P. I. V. Prasad) and the Council of Scientific and Industrial Research (CSIR), India, for the Award of a Senior Research Fellowship.

\section{References}

Brüne, B., von Knethen, A., and Sandau, K.B. (1998) Nitric oxide and its role in apoptosis. Eur $J$ Pharmacol 351: 261-272.

Chan, E.D., Chan, J., and Schluger, N.W. (2001) What is the role of nitric oxide in murine and human host defense against tuberculosis? Current knowledge. Am J Respir Cell Mol Biol 25: 606-612.

Cheruvu, M., Plikaytis, B.B., and Shinnick, T.M. (2007) The acid-induced operon Rv3083-Rv3089 is required for growth of Mycobacterium tuberculosis in macrophages. Tuberculosis (Edinb) 87: 12-20.

Cheung, B.K., Lee, D.C., Li, J.C., Lau, Y.L., and Lau, A.S. (2005) A role for double-stranded RNA-activated protein 
kinase PKR in Mycobacterium-induced cytokine expression. J Immunol 175: 7218-7225.

Choi, B.M., Pae, H.O., Jang, S.I., Kim, Y.M., and Chung, H.T. (2002) Nitric oxide as a pro-apoptotic as well as antiapoptotic modulator. J Biochem Mol Biol 35: 116-126.

Chung, H.T., Pae, H.O., Choi, B.M., Billiar, T.R., and Kim, Y.M. (2001) Nitric oxide as a bioregulator of apoptosis. Biochem Biophys Res Commun 282: 1075-1079.

Cole, S.T., Brosch, R., Parkhill, J., Garnier, T., Churcher, C., Harris, D., et al. (1998) Deciphering the biology of Mycobacterium tuberculosis from the complete genome sequence. Nature 393: 537-544.

Copenhaver, R.H., Sepulveda, E., Armitige, L.Y., Actor, J.K., Wanger, A., Norris, S.J., et al. (2004) A mutant of Mycobacterium tuberculosis $\mathrm{H} 37 \mathrm{Rv}$ that lacks expression of antigen $85 \mathrm{~A}$ is attenuated in mice but retains vaccinogenic potential. Infect Immun 72: 7084-7095.

Cowley, S., Ko, M., Pick, N., Chow, R., Downing, K.J., Gordhan, B.G., et al. (2004) The Mycobacterium tuberculosis protein serine/threonine kinase $\mathrm{PknG}$ is linked to cellular glutamate/glutamine levels and is important for growth in vivo. Mol Microbiol 52: 1691-1702.

Danelishvili, L., McGarvey, J., Li, Y.J., and Bermudez, L.E. (2003) Mycobacterium tuberculosis infection causes different levels of apoptosis and necrosis in human macrophages and alveolar epithelial cells. Cell Microbiol 5: 649-660.

Dao, D.N., Kremer, L., Guerardel, Y., Molano, A., Jacobs, W.R. Jr, Porcelli, S.A., and Briken, V. (2004) Mycobacterium tuberculosis lipomannan induces apoptosis and interleukin-12 production in macrophages. Infect Immun 72: 2067-2074.

Derrick, S.C., and Morris, S.L. (2007) The ESAT6 protein of Mycobacterium tuberculosis induces apoptosis of macrophages by activating caspase expression. Cell Microbiol 9: 1547-1555.

Greenstein, A.E., Macgurn, J.A., Baer, C.E., Falick, A.M., Cox, J.S., and Alber, T. (2007) M. tuberculosis Ser/Thr protein kinase $D$ phosphorylates an anti-anti-sigma factor homolog. PLoS Pathog 3: e49.

Kang, C.M., Abbott, D.W., Park, S.T., Dascher, C.C., Cantley, L.C., and Husson, R.N. (2005) The Mycobacterium tuberculosis serine/threonine kinases PknA and PknB: substrate identification and regulation of cell shape. Genes Dev 19: 1692-1704.

Kaufmann, S.H., and McMichael, A.J. (2005) Annulling a dangerous liaison: vaccination strategies against AIDS and tuberculosis. Nat Med 11: S33-S44.

Long, R., Light, B., and Talbot, J.A. (1999) Mycobacteriocidal action of exogenous nitric oxide. Antimicrob Agents Chemother 43: 403-405.

Lopez, M., Sly, L.M., Luu, Y., Young, D., Cooper, H., and Reiner, N.E. (2003) The 19-kDa Mycobacterium tuberculosis protein induces macrophage apoptosis through Tolllike receptor-2. J Immunol 170: 2409-2416.

Miller, J.H. (1972) Experiment 48. Assay of $\beta$-Galactosidase. In Experiments in Molecular Genetics. Cold Spring Harbor, NY: Cold Spring Harbor Laboratory Press, pp. 352-355.

Molle, V., Girard-Blanc, C., Kremer, L., Doublet, P., Cozzone, A.J., and Prost, J.F. (2003) Protein PknE, a novel transmembrane eukaryotic-like serine/threonine kinase from
Mycobacterium tuberculosis. Biochem Biophys Res Commun 308: 820-825.

Neish, A.S. (2007) TLRS in the gut. II. Flagellin-induced inflammation and antiapoptosis. Am J Physiol Gastrointest Liver Physiol 292: G462-G466.

Papavinasasundaram, K.G., Chan, B., Chung, J.H., Colston, M.J., Davis, E.O., and Av-Gay, Y. (2005) Deletion of the Mycobacterium tuberculosis $\mathrm{pknH}$ gene confers a higher bacillary load during the chronic phase of infection in BALB/c mice. J Bacteriol 187: 5751-5760.

Park, J.S., Tamayo, M.H., Gonzalez-Juarrero, M., Orme, I.M., and Ordway, D.J. (2006) Virulent clinical isolates of Mycobacterium tuberculosis grow rapidly and induce cellular necrosis but minimal apoptosis in murine macrophages. J Leukoc Biol 79: 80-86.

Rao, V., Fujiwara, N., Porcelli, S.A., and Glickman, M.S. (2005) Mycobacterium tuberculosis controls host innate immune activation through cyclopropane modification of a glycolipid effector molecule. J Exp Med 201: 535-543.

Shimono, N., Morici, L., Casali, N., Cantrell, S., Sidders, B., Ehrt, S., and Riley, L.W. (2003) Hypervirulent mutant of Mycobacterium tuberculosis resulting from disruption of the mce1 operon. Proc Natl Acad Sci USA 100: 1591815923.

Singh, A., Singh, Y., Pine, R., Shi, L., Chandra, R., and Drlica, K. (2006) Protein kinase I of Mycobacterium tuberculosis: cellular localization and expression during infection of macrophage-like cells. Tuberculosis (Edinb) 86: 28-33.

Skeiky, Y.A., and Sadoff, J.C. (2006) Advances in tuberculosis vaccine strategies. Nat Rev Microbiol 4: 469-476.

Stafford, S.J., Humphreys, D.P., and Lund, P.A. (1999) Mutations in $d s b A$ and $d s b B$, but not $d s b C$, lead to an enhanced sensitivity of Escherichia coli to $\mathrm{Hg}^{2+}$ and $\mathrm{Cd}^{2+}$. FEMS Microbiol Lett 174: 179-184.

Timm, J., Lim, E.M., and Gicquel, B. (1994) Escherichia colimycobacteria shuttle vectors for operon and gene fusions to lacZ: the pJEM series. J Bacteriol 176: 6749-6753.

Tinsley, C.R., Voulhoux, R., Beretti, J.L., Tommassen, J., and Nassif, X. (2004) Three homologues, including two membrane-bound proteins, of the disulfide oxidoreductase DsbA in Neisseria meningitidis: effects on bacterial growth and biogenesis of functional type IV pili. $J$ Biol Chem 279: 27078-27087.

Tunbridge, A.J., Stevanin, T.M., Lee, M., Marriott, H.M., Moir, J.W., Read, R.C., and Dockrell, D.H. (2006) Inhibition of macrophage apoptosis by Neisseria meningitidis requires nitric oxide detoxification mechanisms. Infect Immun 74: 729-733.

Velmurugan, K., Chen, B., Miller, J.L., Azogue, S., Gurses, S., Hsu, T., et al. (2007) Mycobacterium tuberculosis nuoG is a virulence gene that inhibits apoptosis of infected host cells. PLoS Pathog 3: e110.

Visca, P., Fabozzi, G., Milani, M., Bolognesi, M., and Ascenzi, P. (2002) Nitric oxide and Mycobacterium leprae pathogenicity. IUBMB Life 54: 95-99.

Voskuil, M.I., Schnappinger, D., Visconti, K.C., Harrell, M.I., Dolganov, G.M., Sherman, D.R., and Schoolnik, G.K. (2003) Inhibition of respiration by nitric oxide induces a Mycobacterium tuberculosis dormancy program. J Exp Med 198: 705-713. 
Walburger, A., Koul, A., Ferrari, G., Nguyen, L., PrescianottoBaschong, C., Huygen, K., et al. (2004) Protein kinase G from pathogenic mycobacteria promotes survival within macrophages. Science 304: 1800-1804.

Zhao, Z., Peng, Y., Hao, S.F., Zeng, Z.H., and Wang, C.C. (2003) Dimerization by domain hybridization bestows chaperone and isomerase activities. $J$ Biol Chem 278: 43292-43298.

\section{Supplementary material}

The following supplementary material is available for this article online:

Fig. S1. Confirmation of the pknE deletion in Mycobacterium tuberculosis.

A. PCR: lane $M=100 \mathrm{bp}$ ladder, lane $1=\mathrm{PCR}$ with H37Rv genomic DNA, lane $2=P C R$ with $\Delta p k n E$ genomic DNA, lane $3=$ PCR with complemented $\triangle p k n E$ genomic DNA.

B. Southern blot analysis: Mlul digests of genomic DNA, WT (wild-type), $4.1 \mathrm{~kb}$, indicated by a black arrow; PROBE-EN1 (N-terminal flank of pknE), lanes 1-5: pknE mutants (12.54 kb). Fig. S2. Annexin V/propidium iodide staining of macrophages for detection of apoptosis/necrosis. The representative histograms for day 1 post infection (upper panel, annexin V; lower panel, propidium iodide) with the four categories: control macrophages and macrophages infected with the three strains - H37Rv, pknE mutant and complemented $p k n E$ mutant. The percentage values include both single- and double-stained populations.

Fig. S3. Glutathione assay. GSH levels in lysates taken from THP-1 macrophage cultures infected with H37Rv, pknE mutant and complemented $p k n E$ strains. The assay was conducted according to manufacturer's instructions in triplicates for each sample and the values are graphically represented \pm SEM.

Fig. S4. Cell viability/proliferation assays.

A. Trypan blue dye exclusion for THP-1 macrophages infected with the three strains.

B. MTT (3-[4, 5 dimethylthiazol-2-yl]-2, 5-diphenyl tetrazolium bromide) dye reduction assay for estimation of cell proliferation/ viability: THP-1 macrophages infected with the three strains of M. tuberculosis were subjected to MTT assays at specified time points mentioned above. Triplicate values were recorded and the averages were represented \pm SEM.

Fig. S5. Macrophage survival assay. Briefly, THP-1 macrophages were infected with the three strains of M. tuberculosis, namely wild-type, pknE mutant and complemented mutants. The infected cells were lysed on day 1 , day 3 , day 5 and day 7 post infection, and the diluted lysates were plated on $7 \mathrm{H} 10$ for viable count enumeration. The $y$-axis indicates the colony-forming units (cfu) obtained from the infected cells.

The results are representative of three experiments \pm SEM and were considered statistically significant $\left({ }^{*} P<0.05\right)$.

Table S1A. List of primers and plasmids used for the study. Underlined sequences indicate restriction sites for type II restriction endonucleases.

Table S1B. List of phages and bacterial strains used for the study.

This material is available as part of the online article from: http://www.blackwell-synergy.com/doi/abs/10.1111/ j.1462-5822.2007.01049.x

Please note: Blackwell Publishing is not responsible for the content or functionality of any supplementary materials supplied by the authors. Any queries (other than missing material) should be directed to the corresponding author for the article. 